\title{
Spatio-Temporal Optical Coherence Imaging - a new tool for in vivo microscopy
}

\author{
Maciej Wojtkowski, ${ }^{1}$ Patrycjusz Stremplewski, ${ }^{1,2}$ Egidijus Auksorius, ${ }^{1}$ and Dawid Borycki ${ }^{1, *}$ \\ ${ }^{1}$ Institute of Physical Chemistry, Polish Academy of Sciences, Kasprzaka 44/52 Warsaw, Poland \\ ${ }^{2}$ Institute of Physics, Faculty of Physics, Astronomy and Informatics, Nicolaus Copernicus University, \\ Grudziadzka 5, 87-100 Torun, Poland
}

Received June 12, 2019; accepted June 29, 2019; published June 30, 2019

\begin{abstract}
Optical Coherence Imaging (OCI) including Optical Coherence Tomography (OCT) and Optical Coherence Microscopy (OCM) uses interferometric detection to generate high-resolution volumetric images of the sample at high speeds. Such capabilities are significant for in vivo imaging, including ophthalmology, brain, intravascular imaging, as well as endoscopic examination. Instrumentation and software development allowed to create many clinical instruments. Nevertheless, most of OCI setups scan the incident light laterally. Hence, OCI can be further extended by wide-field illumination and detection. This approach, however, is very susceptible to the so-called crosstalk-generated noise. Here, we describe our novel approach to overcome this issue with spatiotemporal optical coherence manipulation (STOC), which employs spatial phase modulation of the incident light.
\end{abstract}

The main physical limitation of the in vivo microscopic imaging is associated with the light scattering introduced by an irregular and often discontinuous refractive index distribution within a sample. The optical scattering limits the number of image-bearing photons delivered to and received from the sample. As a consequence, the contrast of reconstructed images is reduced dramatically [similarly to a photo made on a foggy day, Fig. 1(b). Trans-]. Another side effect of uneven distribution of the refractive index is a significant image deformation [Fig. 1(b)].

We divide photons traveling through the scattering medium into three categories: ballistic, snake, and multiple- scattered (diffuse photons) [1]. In a turbid medium, most of the detected photons are snake and diffuse, but only ballistic photons carry undisturbed information about the sample. Though we can distinguish different photons by their time-of-flight (TOF), the TOF-gating techniques require expensive short pulse lasers and time-correlated-single-photon-counting or a gated optical image intensifier. Moreover, such devices are sensitive to ambient light, which limits their applicability in real-world microscopic imaging. Interferometric methods, like Optical Coherence Tomography (OCT) [2], or Optical Coherence Microscopy (OCM) [3] solve most of these problems. These methods typically use coherent illumination that leads to speckle formation - strong fluctuations of light intensity caused by mutual interference of mixed transverse modes introduced by light scattering or diffusive reflection. The speckle noise also reduces image quality. Adding all of these effects results in a severe loss of imaging information as it is depicted in Fig. 1(a).

In this manuscript, we present a general idea of novel Spatio-Temporal Optical Coherence (STOC) phase manipulation [4-6]. STOC enables to reduce the contribution from multiply scattered photons by disentangling them from ballistic photons in the interferometric measurement scheme. This disentanglement is achieved by spatial phase modulation of illuminating light and by averaging resulting optical fields or 3-D reconstructions.

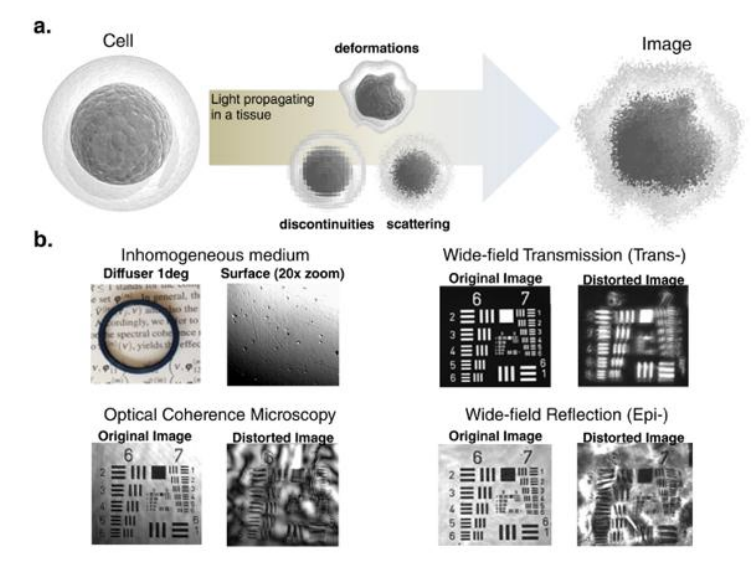

Fig. 1. Graphical representation of image deterioration typical for in vivo studies (a). An example of the controlled experiment of imaging the 1951 USAF resolution test target through $1^{\circ}$ optical diffuser (Wavefront Technology) by Optical Coherence Microscopy (OCM) and wide-field microscopy in Trans- and Epi- modes (b). The diffuser is relatively weak, and distortions are not visible by the naked eye (top left panel in subfigure b) but yet there is a significant resolution loss in Trans- (top right) and also severe deformations in Epi- (bottom right part of subfigure b) microscopic images (incoherent illumination). The diffuser also introduces the speckle pattern in OCM images (coherent illumination).

\footnotetext{
*E-mail: dborycki@ichf. edu.pl
} 


\section{Theoretical description}

The dominant obstacle in cellular level in vivo imaging is the presence of high order distortions introducing intense wavefront modulations. Microand macro-structures of the scattering medium (like blood vessels, fibers, neural or fat cells) are equivalent to uneven, rapid, and random changes in the refractive index distribution along the optical path. These distortions cause delocalization (Fig 2) of points in the resultant image to their original positions in the object. In the case of coherent detection, we can express such delocalization in terms of the crosstalk between transverse modes, which initially compose an undisturbed image.

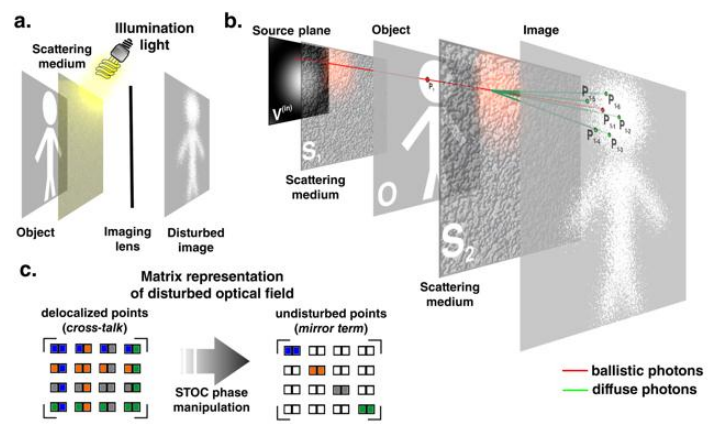

Fig. 2. Imaging through inhomogeneous media in backreflection mode. Light, passing the scattering medium, is reflected from an object, scattered again and then imaged by an optical system (a). In coherent detection, the distorted image is composed of many sets of transverse modes, including one realization corresponding to the original undisturbed image (b). Crosstalk terms may de-correlate once we add transverse modes to illuminate our object - STOC phase manipulation enables us to extract the information about the undisturbed image by mixing and averaging non-diagonal terms in the correlation matrix. The undisturbed image corresponds to the mirror component, which is always distributed diagonally in the correlation matrix (c).

To detect crosstalk-generating transverse modes, we use statistical analysis and matrix notation. According to the theory of linear invariant filters, any linear medium can be represented by a complex-valued transmission (or scattering) matrix, which specifies the mapping between input and output modes. Hence, the effect of optical field propagation through the scattering layers can be represented symbolically as matrix multiplication.

Using the notation from Fig. 2, the column vector $\boldsymbol{V}_{\text {in }}$ represents the optical field emerging from the light source. The linear matrix operator $\boldsymbol{S}_{1}$ modifies this field during light propagation through the first scattering layer. Then, the optical field passes through the object (with matrix $\boldsymbol{O}$ ), and the second scattering layer $\left(\boldsymbol{S}_{2}\right)$. Hence, the output field $\boldsymbol{V}_{\text {out }}$ (the column vector), can be written as

$$
\boldsymbol{V}_{\text {out }}=\boldsymbol{S}_{2} \boldsymbol{O} \boldsymbol{S}_{1} \boldsymbol{V}_{\text {in }}
$$

We see that without scattering layers $\left(\boldsymbol{S}_{1}=\boldsymbol{S}_{2}=\right.$ $\boldsymbol{I}$, where $\boldsymbol{I}$ is the identity matrix), $\boldsymbol{V}_{\text {out }}$ would be equal to $\boldsymbol{O} \boldsymbol{V}_{i n}$. So, the perfect image of the object would be achieved. However, scattering layers break this ideal relation leading to the crosstalk noise. This noise, in turn, scrambles the reconstructed image as sketched in Fig. 2(b).

To overcome this issue, we first quantify the correlation between distinct spatial modes. To estimate these spatial correlations, we observe $\boldsymbol{V}_{\text {out }}$ in time $t$, and then determine the scattering coherency matrix, $\boldsymbol{G}$ defined as:

$$
\boldsymbol{G}=\left\langle\boldsymbol{V}_{\text {out }}(t) \boldsymbol{V}_{\text {out }}^{\dagger}(t)\right\rangle_{t}
$$

where $\langle\cdots\rangle_{t}$ denotes time averaging.

When spatial modes are correlated, the nondiagonal elements of the $\boldsymbol{G}$ matrix will be non-zero [Fig. 2(c)] due to the crosstalk noise. To suppress this noise, we modulate the incident light with a set of deterministic phase patterns, described by another matrix $\boldsymbol{M}$. This modulation yields the altered field $\widetilde{\boldsymbol{V}}_{\text {out }}=\boldsymbol{M} \boldsymbol{V}_{\text {out }}$. After substituting $\widetilde{\boldsymbol{V}}_{\text {out }}$ into Eq. (2) we get the modulated scattering coherency matrix, $\widetilde{\boldsymbol{G}}=\left\langle\boldsymbol{M} \boldsymbol{V}_{\text {out }}(t) \boldsymbol{V}_{\text {out }}^{\dagger}(t) \boldsymbol{M}^{\dagger}\right\rangle_{t}[5]$.

By definition, $\widetilde{\boldsymbol{G}}$ is Hermitian. Hence we can choose $\boldsymbol{M}$ such that it diagonalizes $\widetilde{\boldsymbol{G}}$. If so, we can achieve the ideal mapping between input and output modes by integrating (or averaging) modulated fields (because there will be no cross-talk between different spatial modes). We can perform this averaging in two ways: on the magnitude-basis (intensity averaging) or on the amplitude-basis (complex averaging). Since phase patterns are changing in space and time, we call this approach spatio-temporal optical coherence (STOC) imaging.

\section{STOC based imaging methods and results}

In our method, we assume that mixed terms in $\widetilde{\boldsymbol{G}}$ will de-correlate once we add new sets of transverse modes $\boldsymbol{V}^{(i n)}$ to illuminate object under measurement. In such a case, averaging of many fields scattered from the object illuminated with the set of transverse modes will enable averaging out non-diagonal elements of $\widetilde{\boldsymbol{G}}$ and retrieve undisturbed information about the object. When output fields $\boldsymbol{V}_{\text {out }}$ are measured in the Fourierdomain, we can interpret each entry of $\widetilde{\boldsymbol{G}}$ matrix, after normalization, as the complex spectral degree of coherence (correlation matrix). The diagonalization process will correspond to the decorrelation of crosstalk terms [Fig. 2(c)] and can be, in practice, introduced by dynamic spatial phase modulation of light incident on the sample. In practice, the final implementation of this idea requires an appropriately designed spatial phase modulator for a particular application and correct synchronization of the detection system. Such instrumentation would be easily adaptable to any 
existing digital- optical device, including wide-field microscopes, OCT, OCM, holographic imaging,

a.

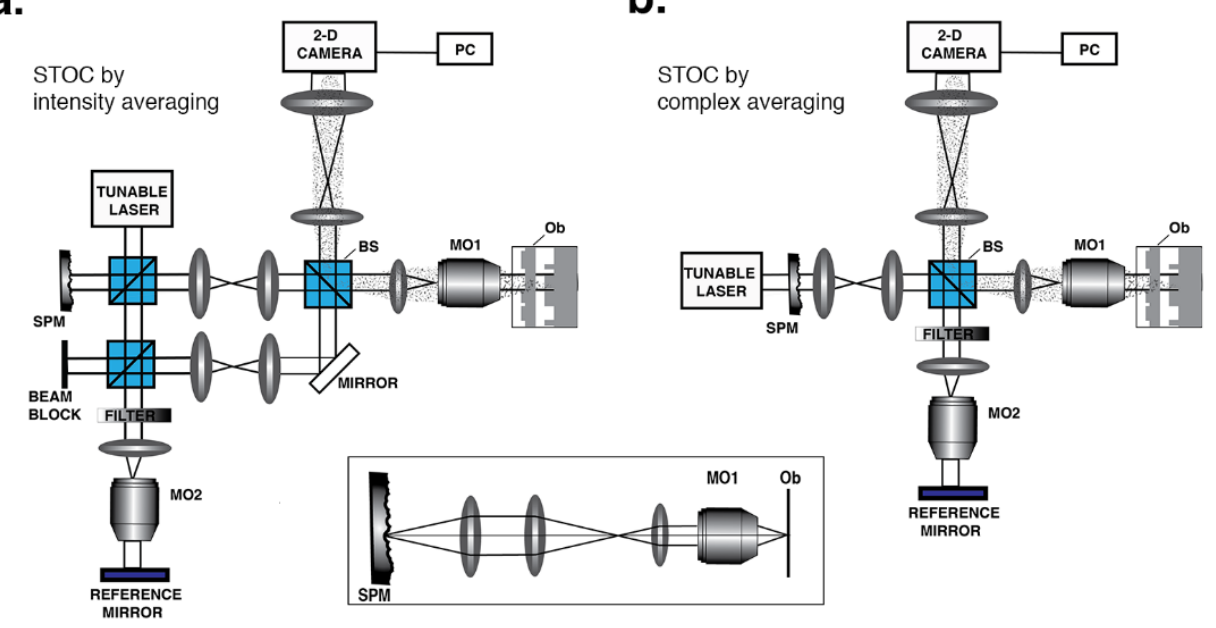

quantitative phase microscopy, etc.

b.

Fig. 3. Two optical setups for STOC imaging for (a) magnitude-based averaging and (b) amplitude-based averaging; BS - beam splitter, MO - microscopic objective, $\mathrm{Ob}$ - object, SPM -Spatial Phase Modulator.

So far, we have identified two optimal, hardwarebased ways of tailoring correlation matrix $\widetilde{\boldsymbol{G}}$ to remove non-diagonal terms and to reject multiply scattered light (Fig. 3). These two techniques differ by the implemented averaging method required to reduce the effects of light scattering. The core of both of them is Fourier-domain Full-Field OCT (Fd-FF-OCT) imaging set-up [7-12]. Fd-FF-OCT enables the acquisition of complex data with coherence gating that provides optical axial sectioning [13]. Fd-FF-OCT also allows for applying multimodal transverse illumination and detection [12]. Those three parameters are critical for STOC implementation in biomedical imaging.

\section{Method No 1. STOC by intensity averaging}

In the first possible, STOC materializations the Spatial Phase Modulation [SPM in Fig. 3(a)] is introduced by a liquid crystal Spatial Light Modulator (SLM $-1920 \times 1080$ pixels, $8 \mu \mathrm{m}$ pixel size, $30 \mathrm{~Hz}$ phase switching rate, PLUTO NIR2, HoloEye). The SPM is acting only on the sample arm of the Mach-Zehnder interferometer (MZI), which employs a tunable semiconductor light source (BroadSweeper BS-840-2, Superlum) that can be tuned in the range of $800-878 \mathrm{~nm}$. The active surface of the SLM is imaged on the sample plane by a lens system and a microscope objective. Such an optical arrangement provides conjugation between the phase modulation plane and the sample plane. Each phase mask is projected on the sample for the period that it takes for the tunable laser (BroadSweeper) to be tuned through the entire spectral range. CMOS camera registers interferometric images for each optical frequency emitted from the light source. This technique has been recently described in detail by Borycki et al. [5]. In this paper, we demonstrated that in order to assure phase masks decorrelation and diagonalization of the correlation matrix $\widetilde{\boldsymbol{G}}$, it is sufficient to use a set of $M$ phase masks with a random spatial phase distribution varying between 0 and $2 \pi$. Then the final, crosstalk-free reconstruction of an object is obtained simply by averaging intensity representations of the volumetric Fd-FF-OCT data.

Figure 4 shows the data processing scheme required to analyze the measured data. The first step of signal processing is similar to any Fd-FFOCT technique [14]. First, we convert the registered spectral fringe patterns (optical frequency, $\omega$-domain) to volumetric representation (depth-resolved, z-domain) of complex backscattered optical fields. To this end, we calculate the Fast Fourier Transformation (FFT) for each pixel separately. Subsequently, we extract a single axial plane representing the layer of interest. This plane yields intensity and phase maps, each of which has $N \times N$ pixels (step I in Fig. 4). To ensure that averaging will be useful we check if phase patterns diagonalize the $N^{2} \times N^{2}$ correlation matrix $\widetilde{\boldsymbol{G}}$ [step II in Fig. 4]. To do so, we calculate pixel-wise normalized $N \times N$ crosscorrelation matrix of complex images acquired for consecutive phase masks $(m=1, \ldots, M)$. We then remap the resultant matrix to a vector, containing $N^{2}$ elements. This vector becomes the nth row (out of total $N^{2}$ rows) of the scattering coherency matrix. Finally, if matrix $\widetilde{\boldsymbol{G}}$ is diagonal, 
we average $M$ intensity images to obtain the final image of the axial plane [step III in Fig. 7].

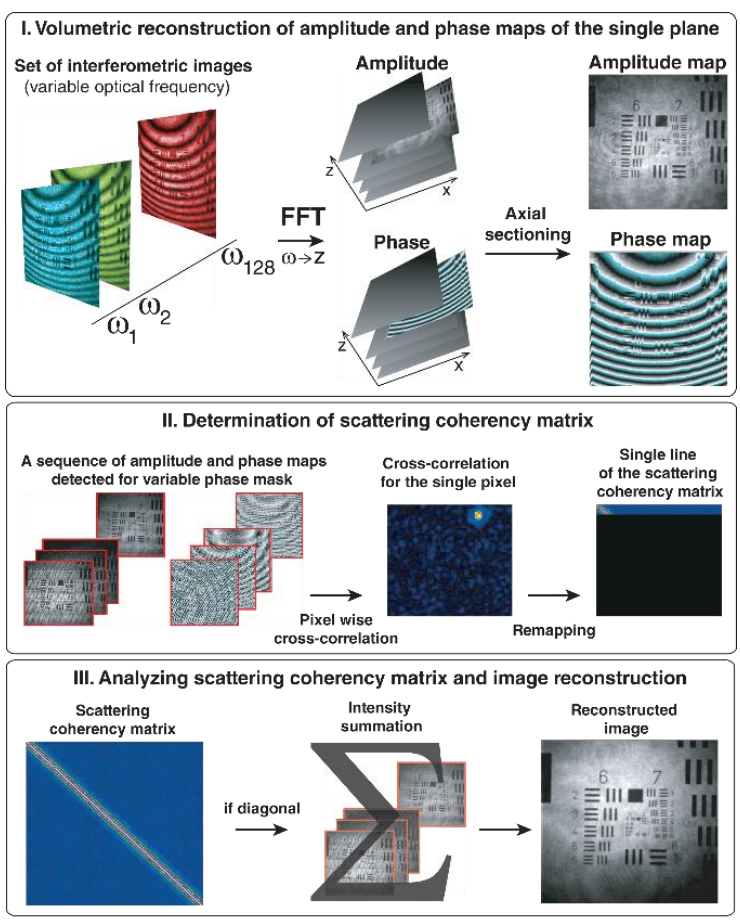

Fig. 4. Data processing scheme required to understand and optimize crosstalk removal.
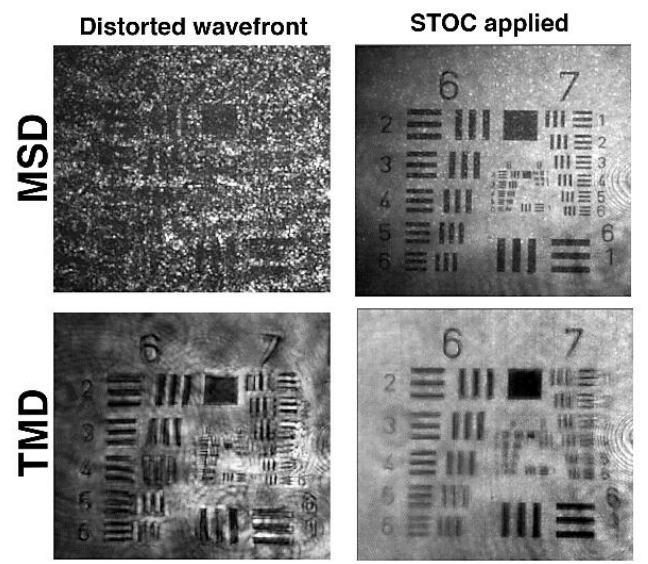

Fig. 5. Image distortion removal by spatio-temporal optical coherence manipulation with intensity averaging. 1951 USAF test target is imaged through two diffusers (two separate experiments): the TMD and MSD (see text). MSD introduces strong scattering and resulting speckle noise, whereas the TMD induces relatively weak wavefront deformations. Modulation of the incident light by random phase masks enables to diagonalize the scattering coherency matrix so that aberrations and speckle noise is suppressed after the summation of intensity images is carried out.

To validate this approach, we use the microspheres diffuser (MSD) and a tailored glass micro diffuser (TMD) with a $1^{\circ}$ specular scattering angle [the same as used to obtain data from Fig. 1(b)]. MSD consists of $1 \mu \mathrm{m}$ diameter aluminum spheres (Premasol DA Alumina Suspension, $\mathrm{Al}_{2} \mathrm{O}_{3}$ powder) solution sandwiched between two cover-slip glasses, forming $25 \mu \mathrm{m}$-thick suspension. Using the collimated transmission method, we estimated the optical thickness (OT) of the MSD to be eight mean free paths $\left(\mathrm{OT}=81_{\mathrm{s}}\right)$. Each diffuser was placed on the 1951 USAF resolution test target and imaged with the setup shown in Fig. 3(a). Resulting crosssectional images are compared to the undistorted case (without diffuser) in Fig. 5. Both diffusers introduced strong wavefront modulations resulting in image distortions (left column in Fig. 5). To suppress the coherent crosstalk noise generated by the TMD and the MSD we applied STOC manipulation with $M=512$ random phase masks. The speckle noise generated by the MSD has been suppressed, revealing high-resolution features of the sample [MSD row in Fig. 5]. Similarly, distorted and shifted shapes and the locations of bars and digits of the resolution chart were successfully restored to the original position after enabling STOC manipulation [TMD row in Fig. 5].

\section{Method No 2. STOC by complex averaging}

The second STOC realization has been recently described by our group [6]. The method involves the recording of tens or hundreds of interferometric fringe patterns, resulting from the reflection or backscattering of the light from the object and the reference mirror, by a high-speed 2-D camera. Each of these fringe patterns is acquired while the phase of light entering the interferometer is spatially modulated [Fig. 3(b)]. The modulation can be performed during the camera exposure time. In this way, many interferometric signals resulting from the spatial phase modulation are averaged in a complex manner. Since this averaging takes place during the opening of the CMOS camera shutter (complex averaging) the phase relations in the beams from the reference arm and the object arm of the interferometer are retained only for those waves that do not undergo strong distortions of the wavefront in one of the interferometer arms. Distorted waves, which are responsible for the crosstalk effect, interfere with variable phases, meaning that the visibility of the interferometric fringes from these waves is washed-out. The recording of interference patterns is repeated for each wavelength provided by the laser and followed by the reconstruction of a three-dimensional structure of the object through Fourier Transform.

A vital element of the optical system depicted in Fig. 3(b) as a Spatial Phase Modulator (SPM) is Dyoptyka's phase-randomizing deformable membrane (DM) with a unique design. The membrane vibrates at a set of frequencies above $200 \mathrm{kHz}$. Likewise in the previous method, a rapidly tunable laser is employed (BroadSweeper, Superlum). The membrane is imaged on the sample and the reference mirror by identical optics. Images of the reference mirror and the object $\mathrm{Ob}$ are 
formed on a high-speed Photron FastCam CMOS camera. Interferometric images are recorded by a CMOS camera as a function of a wavelength that is changed during the sweep of the tunable laser. The phase is spatially modulated by the membrane fluctuations in a time shorter than the exposure time of the CMOS camera.

\section{STOC by complex averaging enables high-speed in} vivo imaging

In order to demonstrate the rejection of multiplescattered photons, we used a phantom consisting of two scattering layers immersed in a transparent material as object $\mathrm{Ob}$. One scattering layer with a thickness of about 150 microns is above the second layer, the thickness of which is a few millimeters. There are two "IChF" logotypes on the surface of the scattering layers that are slightly shifted in XY. Details of phantom properties and its fabrication can be found in the paper by Stremplewski et al. [6] Representational results are depicted in Fig. 6. Both cross-sections: along XY and XZ planes, show improvement of image quality due to crosstalk rejection. This technique was also applied for in vivo skin imaging.

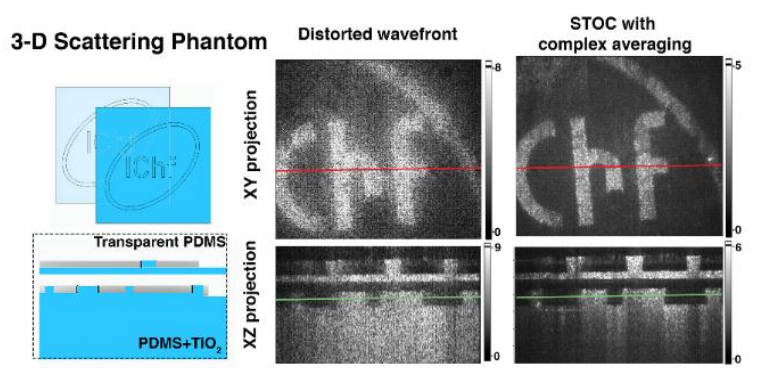

Fig. 6. Spatio-temporal optical coherence manipulation with complex averaging suppresses crosstalk in volumetric reconstructions of the scattering phantom. The red line indicates the location of the $\mathrm{XZ}$ plane displayed in the bottom row and the green line corresponds to the location of the $\mathrm{XY}$ images displayed above.

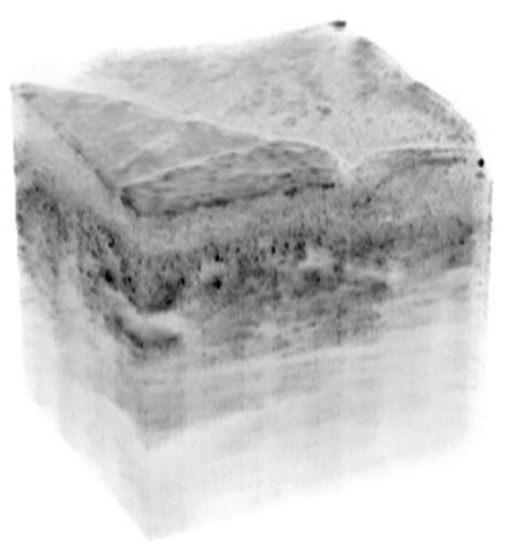

Fig. 7. 3-D rendering of complex averaging STOC method data acquired for human forearm skin in vivo (volume size $1 \times 1 \times 1 \mathrm{~mm}^{3}$ ).
Figure 7 shows a three-dimensional rendering of Fd-FF-OCT data with implemented STOC and complex averaging. The reductions of distortions and scattering both reveal anatomical details and enable differentiation of the tissue structures. The stratum corneum, the stratum granulosum, and the stratum spinosum layers - all are distinguishable clearly in our reconstructions. The stratum spinosum with a layer of polyhedral keratinocytes is particularly well visible as a granular layer. The acquisition time of the full data set was $0.4 \mathrm{~s}$.

In this paper, we have presented spatio-temporal optical coherence (STOC) modulation that can be used to suppress image distortions from optical inhomogeneities in the Fourier domain Full Field OCT (Fd-FF-OCT).

We have shown that sequential phase modulation, synchronized with light acquisition decorrelates the coherent noise from spatial crosstalk. The efficiency of crosstalk suppression is quantified with the scattering coherency matrix. Next, we have presented that by integrating image intensities or complex amplitudes, we can improve Fd-FF-OCT imaging through scattering phantoms and in vivo human forearm skin imaging. Importantly, our approach does not rely on any prior knowledge of the inhomogeneous medium. We effectively synthesized the spatially incoherent detection (magnitude-based averaging) or improved confocal gating (amplitude-based averaging).

The crosstalk is due to high spatial coherence. So, this problem is not present in conventional Timedomain (Td-) FF-OCT systems that rely on spatially incoherent illumination [12]. Thus, our approach (primarily that based on magnitude averaging) should provide comparable results as Td-FF-OCT. However, conventional FF-OCT systems are slower than Fd-FF-OCT. Hence, these improvements in speed performance allow achieving lateral phase stability that can be beneficial for numerical phase correction to improve STOC imaging further.

We are currently investigating the differences between two STOC implementations and expect to publish these results shortly.

We acknowledge the funding from National Science Center (NCN, 2016/22/A/ST2/00313) and European Union's Horizon 2020: research and innovation programme (666295).

\section{References}

[1] L. Wang, P.P. Ho, C. Liu, G. Zhang, R.R. Alfano, Science 253 , 769 (1991).

[2] D. Huang, E.A. Swanson, C.P. Lin, J.S. Schuman, W.G. Stinson, W. Chang, M.R. Hee, T. Flotte, K. Gregory, C.A. Puliafito, et al., Science 254, 1178 (1991).

[3] J. A. Izatt, E. A. Swanson, J. G. Fujimoto, M. R. Hee, and G. M. Owen, Opt. Lett. 19, 590 (1994).

[4] D. Borycki, M. Nowakowski, M. Wojtkowski, Opt. Lett. 38, 4817 (2013). 
[5] D. Borycki, M. Hamkalo, M. Nowakowski, M. Szkulmowski, M. Wojtkowski, Biomed. Opt. Express 10, 2032 (2019).

[6] P. Stremplewski, E. Auksorius, P. Wnuk, L. Kozon, P. Garstecki, M. Wojtkowski, Optica 6, 608 (2019).

[7] L. Vabre, A. Dubois, A.C. Boccara, Opt. Lett. 27, 530 (2002).

[8] M. Laubscher, M. Ducros, B. Karamata, T. Lasser, R. Salathé, Opt. Express 10, 429 (2002).

[9] A. Dubois, A.C. Boccara, Full-Field Optical Coherence Tomography (Springer Berlin Heidelberg, Berlin, Heidelberg, 2008), pp. 565-591.

[10] O. Thouvenin, K. Grieve, P. Xiao, C. Apelian, A.C. Boccara, Biomedical Opt. Express 8, 622 (2017).

[11] F. Fercher, C.K. Hitzenberger, M. Sticker, E. MorenoBarriuso, R. Leitgeb, W. Drexler, H. Sattmann, Optics Commun. 185, 57 (2000).

[12] R.A. Leitgeb, Biomed Opt Express 10, 2177 (2019).

[13] J. Fujimoto W. Drexler, Introduction to Optical Coherence Tomography (Springer, Berlin, Heidelberg, 2008), pp. 1-45.

[14] J.A. Izatt, M.A. Choma, A.-H. Dhalla, Theory of Optical Coherence Tomography (Springer International Publishing, Cham, 2015), pp. 65-94. 